\title{
Long-term survival after repeated resection for lung metastasis originating from pancreatic cancer: a case report
}

\author{
Yasunori Uesato ${ }^{1 *}$, Koichi Tamashiro ${ }^{2}$ and Mitsuhisa Takatsuki ${ }^{1}$
}

\begin{abstract}
Background: Pancreatic cancer has a grave prognosis. Most patients with metastatic pancreatic cancer are inoperable, and case reports of resection of lung metastasis from pancreatic cancer are rare. This patient underwent resection of a lung metastasis twice after pancreaticoduodenectomy for pancreatic cancer.

Case presentation: A 75-year-old man with pancreaticoduodenectomy and adjuvant chemotherapy for pancreatic cancer was diagnosed with a lung metastasis 48 months after surgery. Histological findings after thoracoscopic partial resection of the right lung by video-assisted thoracic surgery confirmed the presence of a lung metastasis originating from the pancreatic cancer. The patient refused chemotherapy. A new lung metastasis was detected 84 months following the second surgery (132 months after the pancreaticoduodenectomy). After thoracoscopic partial resection of the left lung by video-assisted thoracic surgery, the histological findings once again confirmed a metastasis that originated from the pancreatic cancer. The patient refused chemotherapy and remained alive and relapse-free after the 10-month follow-up.
\end{abstract}

Conclusion: Detection and resection of an isolated lung metastasis originating from pancreatic cancer may improve prognosis. Careful follow-up may be warranted to identify patients who might benefit from aggressive local treatment of oligometastasic pancreatic cancer.

Keywords: Pancreatic cancer, Repeated resection of lung metastasis, Prognosis, Long-term survival

\section{Background}

Pancreatic cancer has a very poor prognosis and high mortality. Patients with metastatic pancreatic cancer have a 5 -year overall survival of only $2 \%$ [1], and less than $5 \%$ of patients who receive chemotherapy for advanced pancreatic cancer survive for more than 5 years [2-5]. Distant metastasis of pancreatic cancer mostly occurs in the liver followed by lungs, regional lymph nodes, and the peritoneum [6]. Successful surgical intervention in patients with an isolated lung recurrence of pancreatic cancer has been reported [7-11], but cases involving multiple pulmonary resection of pancreatic

\footnotetext{
* Correspondence: h116638@med.u-ryukyu.ac.jp

'Department of Digestive and General Surgery, University of Ryukyus, 207

Uehara, Nishihara, Okinawa, Japan

Full list of author information is available at the end of the article
}

cancer metastasis are very rare. This patient underwent two separate lung resections for pancreatic cancer metastasis with good long-term survival.

\section{Case presentation}

The patient was a 75-year-old man with a pancreatic tumor that was detected by ultrasonography during a routine medical checkup. Computed tomography (CT) revealed a mass in the head of the pancreas that was diagnosed as pancreatic cancer. Evidence of distant metastasis was not found by CT. The patient's CEA (1.1 ng/ $\mathrm{ml})$ and CA19-9 $(8.0 \mathrm{U} / \mathrm{ml})$ were within their normal ranges. The histopathological diagnosis following pancreaticoduodenectomy (PD) with clear margins confirmed a well-differentiated, invasive adenocarcinoma, pT2 $(25 \times 32 \mathrm{~mm}), \mathrm{N} 2, \mathrm{M} 0$, ly3, v2, pStageIII using the

\section{Springer Open}

(c) The Author(s). 2020 Open Access This article is licensed under a Creative Commons Attribution 4.0 International License, which permits use, sharing, adaptation, distribution and reproduction in any medium or format, as long as you give appropriate credit to the original author(s) and the source, provide a link to the Creative Commons licence, and indicate if changes were made. The images or other third party material in this article are included in the article's Creative Commons licence, unless indicated otherwise in a credit line to the material. If material is not included in the article's Creative Commons licence and your intended use is not permitted by statutory regulation or exceeds the permitted use, you will need to obtain permission directly from the copyright holder. To view a copy of this licence, visit http://creativecommons.org/licenses/by/4.0/. 
eighth edition UICC classification system criteria. Adjuvant chemotherapy with tegafur, gimeracil, and oteracil potassium (100 mg/day) was given for 1 year post-PD. At the 48-month follow-up, a new $5 \times 3$-mm lesion in the lower right lobe of the lung was detected by CT (Fig. 1). It was a new lesion suggestive of malignant disease, and metastatic lung cancer was suspected because it was an isolated solid mass. The patient's CEA $(1.2 \mathrm{ng} / \mathrm{ml})$ and CA19-9 $(5.0 \mathrm{U} / \mathrm{ml})$ concentrations were normal, and other metastases were not detected by CT. Histopathological findings of the lung lesion following thoracoscopic partial resection of the right lung by videoassisted thoracic surgery (VATS) revealed a welldifferentiated tumor with irregular growth of atypical gland ducts showing irregular flexion and bifurcation characteristic of a mucus-producing adenocarcinoma. Immunostaining findings were consistent with a lung metastasis of pancreatic cancer rather than a primary lung cancer (Fig. 2). Adjuvant chemotherapy was recommended to prevent recurrence because the lung metastasis was suspected to be pancreatic cancer based on the clinical course, but the patient refused.

Eighty-four months after the first pulmonary resection (132 months after PD), CT and positron emission tomography (PET) revealed a new $10 \times 10$-mm lesion in the lower lobe of the left lung (Fig. 3). A lung metastasis was suspected because the mass was new, isolated, solid, and had smooth margin. However, pleural invagination was also found, suggesting primary lung cancer. A bronchoscopy for a histopathological diagnosis was not performed because of the tumor location. CT and PET did not find

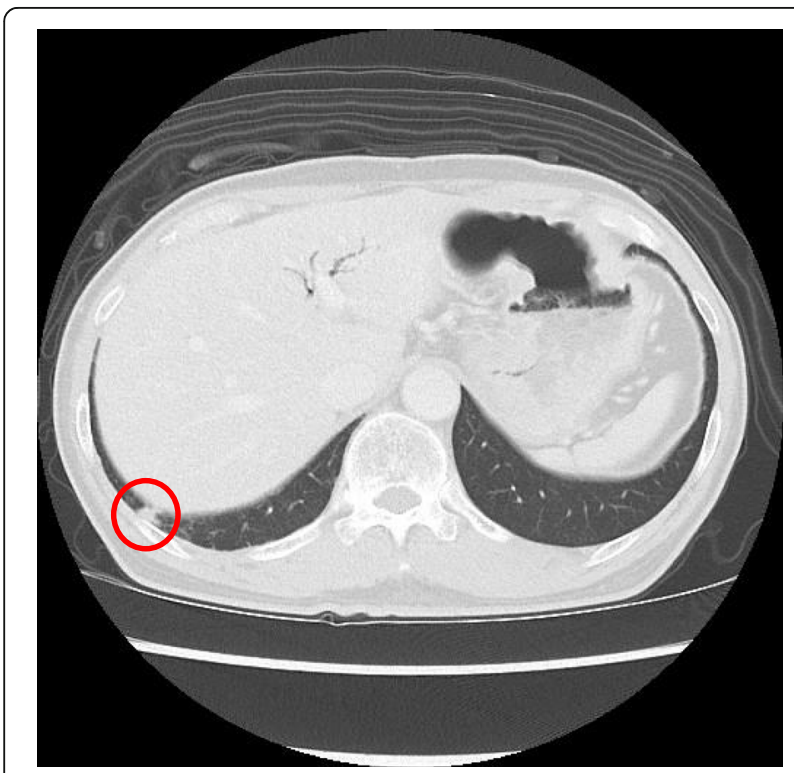

Fig. 1 Computed tomography (CT) shows a $5 \times 3$-mm tumor mass near the base of the right lung. This was a new isolated, localized, solid lesion other metastases. Serum CA19-9 was high $(90 \mathrm{U} / \mathrm{ml}$, normal $<37 \mathrm{U} / \mathrm{ml}$ ). VATS was performed and was followed by an uneventful postoperative recovery without severe complications and a return of the CA19-9 concentration to normal levels. The histopathological findings were similar to those for the first lung lesion, finding a well-differentiated adenocarcinoma that was diagnosed as a pancreatic cancer metastasis (Fig. 4). The patient refused adjuvant chemotherapy and had experienced no severe complications or relapse at the 10month follow-up.

\section{Discussion}

Generally, patients with recurrent pancreatic cancer are indicated for chemotherapy or best supportive care, and not for surgical intervention. The prognosis is extremely poor for recurrence in the liver even if the lesion is solitary [12], but surgical intervention for solitary recurrence in the lung has been reported to improve prognosis [7-11]. Downs-Canner et al. reported that surgery for lung recurrences prolonged overall survival compared with chemotherapy or best supportive care [13]. Our patient remained alive and recurrence-free 142 months after PD, which is far longer than expected for stage III pancreatic cancer.

Previous reports of surgical intervention for pancreatic cancer recurrence in the lung are summarized in Table 1. The mean time to pulmonary resection was 26 months. Most patients had one lung recurrence, and the mean overall survival after lung resection was 34 months. As in this patient, the time to subsequent recurrence was prolonged, and overall survival was improved, by pulmonary resection. Only one previous report included multiple resection procedures, and in line with our experience, the patient also had long-term survival of 8 years after surgery. The available evidence indicated that pulmonary resection for recurrence can significantly prolong survival in selected patients.

Distinguishing between primary and metastatic lung cancer is needed to guide treatment. Thyroid transcription factor (TTF)-1 expression has been described as a useful marker of primary lung cancer and highly specific for primary lung adenocarcinoma [14, 15]. TTF-1 immunostaining was negative in this patient. However, because TTF-1 positivity is reported to be low in mucinous bronchioloalveolar carcinoma, it was not conclusive in this case of mucin-producing adenocarcinoma [16]. Cytokeratin (CK)7 and CK20 are useful for distinguishing between lung primary and metastatic tumors. Primary lung cancer has many CK-positive and CKnegative patterns, which are not consistent with the findings of this case, but there are reports of CK7- andCK20-positive mucinous bronchioloalveolar carcinomas $[17,18]$. Napsin A has been reported to be specific for primary lung adenocarcinoma and was negative in this 

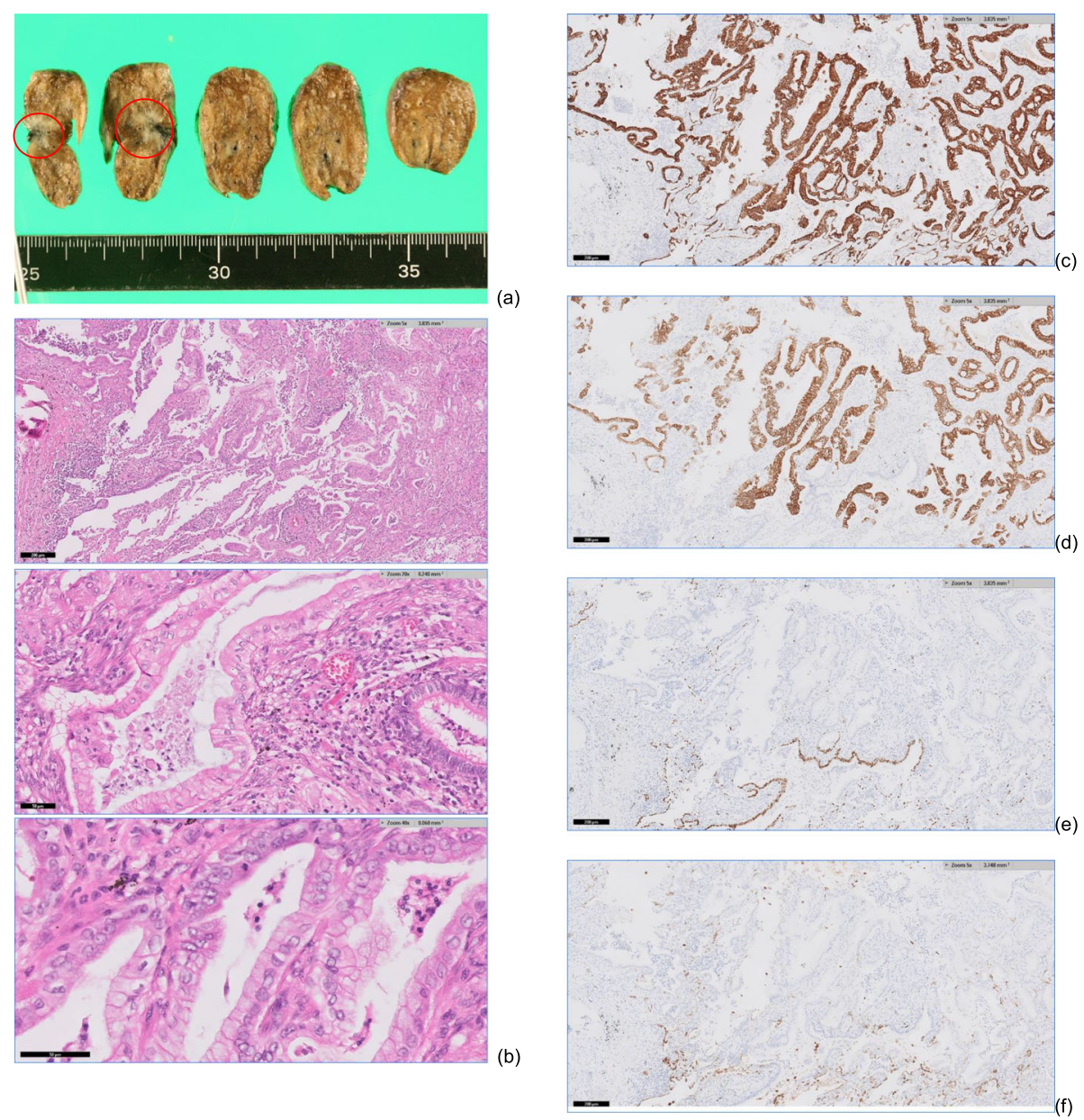

Fig. 2 Histopathological and immunostaining findings with $\mathbf{a}$ the tumor location indicated by the circle and $\mathbf{b}$ hematoxylin-eosin staining of tissue with columnar tumor cells, nuclear atypia, and mucus containing mucus vesicles. Immunostaining shows $\mathbf{c}$, $\mathbf{d}$ diffuse expression of cytokeratin 7 and 20 and $\mathbf{e}, \mathbf{f}$ negative staining of thyroid transcription factor 1 and Napsin A

case [19]. Although it was difficult to completely rule out primary lung cancer in this patient by histopathology and immunostaining, the findings were consistent with metastatic lung cancer and were also consistent with the clinical course.

The available evidence of the effectiveness of metastatic resection of pulmonary metastasis of pancreatic cancer is not sufficient to make an overall recommendation. Surgical indications must be carefully considered on a case-by-case basis. The following criteria may help to determine whether surgery is indicated: the patient can tolerate surgery, the primary lesion is controlled, other metastases are not present outside of the lung, and all lung metastases can be resected. This patient and most of the patients in the reports described in Table 1 met those criteria. The overall experience indicates that the survival of patients with a metachronous solitary tumor recurrence and a long disease-free interval following resection of the primary tumor could be prolonged by surgical intervention. This patient met the suggested conditions at the time of the first and second lung resections and experienced prolonged postoperative survival.

It is not clear why the prognosis of patients with metachronous lung recurrence is improved by surgery. Wangjam et al. and Takano et al. suggested that lung recurrences may have a tumor microenvironment different from that of the primary tumor $[12,20]$. Kurahara et al. reported that pancreatic cancer cells that form a solitary lung lesion may be less aggressive than those that form multiple lung metastases or metastasize to other organs [11]. A better understanding of the mechanisms underlying differences in metastatic behavior would increase the accuracy of prognosis and help to determine surgical indications for lung recurrence.

\section{Conclusions}

Lung resection twice for metachronous lung metastasis resulted in long-term recurrence-free survival after PD 

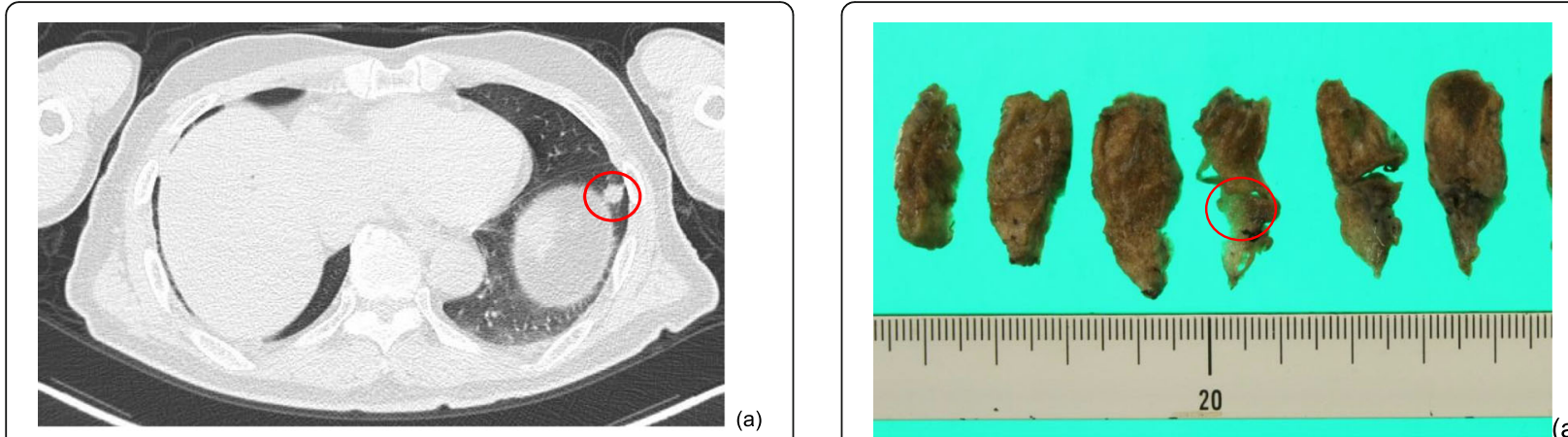

(a)

20

(a)

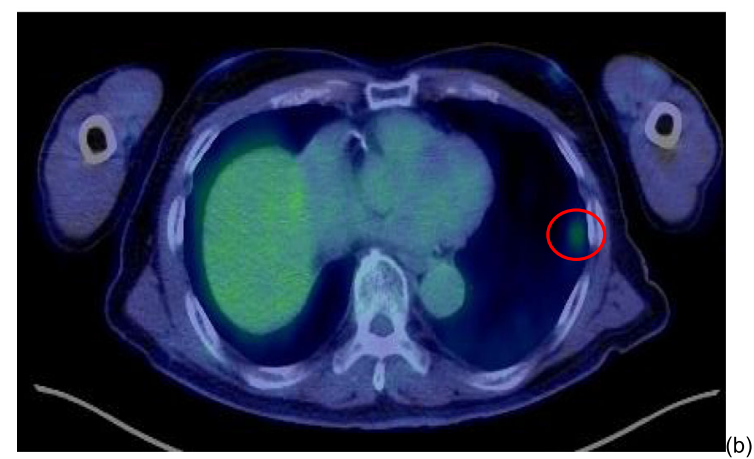

Fig. 3 Computed tomography $(\mathrm{CT})$ and positron emission tomography (PET) show a $10 \times 10-\mathrm{mm}$ tumor in the left lung S8 (a). This was a new isolated, localized, and solid lesion. Lung cancer metastasis was suspected but pleural indentation was also present, suggesting the possibility of primary lung cancer. PET shows strong uptake of fluorodeoxyglucose at the same site, suggesting lung metastasis (b)

Fig. 4 Histopathological findings with a the tumor location

indicated by the circle and $\mathbf{b}$ hematoxylin-eosin stained tissue with alveolar replacement and proliferation of atypical gland ducts with irregular mucus-containing branches 


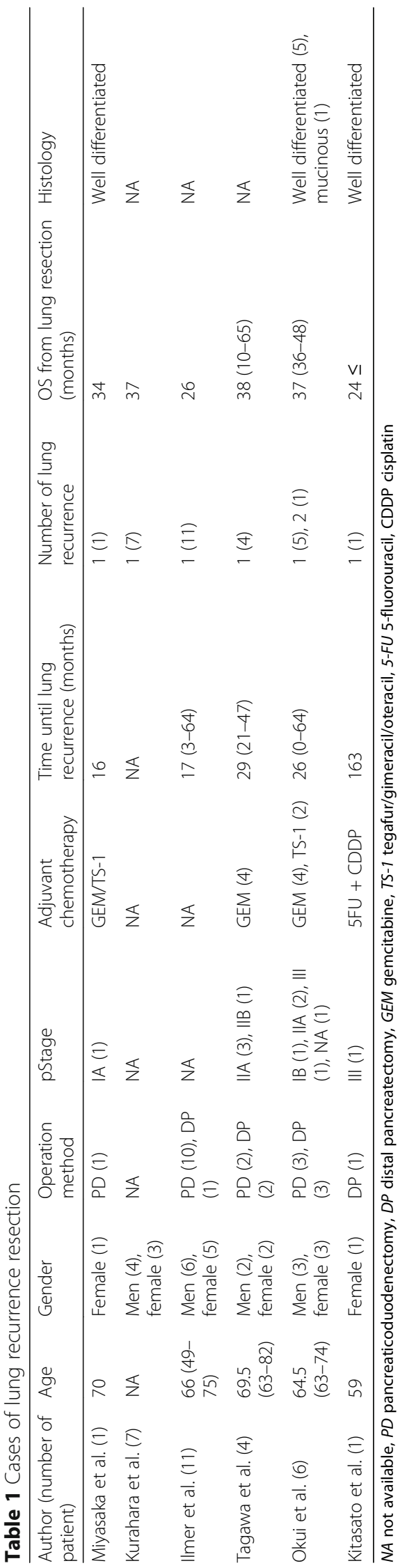


for pancreatic cancer. Rare occurrences of solitary pancreatic cancer metastasis to the lung may be curable with aggressive resection, but the criteria for surgical selection are not clear. Future studies may identify prognostic biomarkers that would help to identify patients who would benefit from surgical intervention, similar to the patient described here.

\section{Abbreviations}

CT: Computed tomography; PD: Panceraticoduodenectomy; PET: Positron emission tomography; VATS: Video-assisted thoracic surgery;

TTF: Transcription factor; CK: Cytokeratin

\section{Acknowledgements}

We thank all the other members and staff for their contributions to the completion of our case report. The authors would like to thank Enago (www. enago.jp) for the English language review.

\section{Authors' contributions}

All authors contributed to the acquisition and analysis of data. YU was a major contributor in writing the manuscript. The authors read and approved the final manuscript.

\section{Funding}

No funding was obtained from the private or public sector for this research.

\section{Availability of data and materials}

Presented within the manuscript. Please contact the author for additional data requests.

\section{Ethics approval and consent to participate}

Obtained from the patient in written form.

\section{Consent for publication}

Witten consent to publish was obtained for the publication of all clinical details and images, and the consent form is available for review by the editor of the journal.

\section{Competing interests}

The authors declare that they have no competing interests.

\section{Author details}

'Department of Digestive and General Surgery, University of Ryukyus, 207 Uehara, Nishihara, Okinawa, Japan. ${ }^{2}$ Department of Pathology, University of Ryukyus, 207 Uehara, Nishihara, Okinawa, Japan.

Received: 8 March 2020 Accepted: 1 April 2020

Published online: 07 April 2020

\section{References}

1. Conroy T, Bachet JB, Ayav A, Huguet F, Lambert A, Caramella C, et al. Current standards and new innovative approaches for treatment of pancreatic cancer. Eur J Cancer. 2016;57:10-22.

2. Shi S, Yao W, Xu J, Long J, Liu C, Yu X. Combinational therapy: new hope for pancreatic cancer? Cancer Lett. 2012;317(2):127-35.

3. Neoptolemos JP, Kleeff J, Michl P, Costello E, Greenhalf W, Palmer DH. Therapeutic developments in pancreatic cancer: current and future perspectives. Nat Rev Gastroenterol Hepatol. 2018;15(6):333-48.

4. Conroy T, Hammel P, Hebbar M, Ben Abdelghani M, Wei AC, Raoul JL, et al. FOLFIRINOX or gemcitabine as adjuvant therapy for pancreatic cancer. N Engl J Med. 2018:379(25):2395-406.

5. Buscail L. Commentary: pancreatic cancer: is the worst to come? Int J Epidemiol. 2017:46(6):1774-5

6. Katz MH, Wang H, Fleming JB, Sun CC, Hwang RF, Wolff RA, et al. Long-term survival after multidisciplinary management of resected pancreatic adenocarcinoma. Ann Surg Oncol. 2009;16(4):836-47.

7. Miyasaka M, Noji T, Ohtaka K, Chiba R, Sato S, Shoji Y, et al. Long-term survival after repeated resection of metachronous lung metastases from
pStage IA pancreatic adenocarcinoma. Clin J Gastroenterol. 2018;11(1):5361.

8. Okui M, Yamamichi T, Asakawa A, Harada M, Horio H. Resection for pancreatic cancer lung metastases. Korean J Thorac Cardiovasc Surg. 2017. 50(5):326-8.

9. Tagawa T, Ito K, Fukuzawa K, Okamoto T, Yoshimura A, Kawasaki T, et al. Surgical resection for pulmonary metastasis from pancreatic and biliary tract cancer. Anticancer Res. 2017;37(3):1413-6.

10. Ilmer M, Schiergens TS, Renz BW, Schneider C, Sargut M, Waligora R, et al. Oligometastatic pulmonary metastasis in pancreatic cancer patients: safety and outcome of resection. Surg Oncol. 2019:31:16-21.

11. Kurahara H, Maemura K, Mataki Y, Tanoue K, lino S, Kawasaki Y, et al. Lung recurrence and its therapeutic strategy in patients with pancreatic cancer. Pancreatology. 2020;20(1):89-94.

12. Wangiam T, Zhang Z, Zhou XC, Lyer L, Faisal F, Soares KC, et al. Resected pancreatic ductal adenocarcinomas with recurrence limited in lung have a significantly better prognosis than those with other recurrence patterns. Oncotarget. 2015;6(34):36903-10

13. Downs-Canner S, Zenati M, Boone BA, Varley PR, Steve J, Hogg ME, et al. The indolent nature of pulmonary metastases from ductal adenocarcinoma of the pancreas. J Surg Oncol. 2015;112(1):80-5.

14. Nakamura N, Miyagi E, Murata S, Kawaoi A, Katoh R. Expression of thyroid transcription factor-1 in normal and neoplastic lung tissues. Mod Pathol. 2002:15(10):1058-67.

15. Moldvay J, Jackel M, Bogos K, Soltesz I, Agocs L, Kovacs G, et al. The role of TTF-1 in differentiating primary and metastatic lung adenocarcinomas. Pathol Oncol Res. 2004;10(2):85-8.

16. Saad RS, Liu YL, Han H, Landreneau RJ, Silverman JF. Prognostic significance of thyroid transcription factor-1 expression in both early-stage conventional adenocarcinoma and bronchioloalveolar carcinoma of the lung. Hum Pathol. 2004:35(1):3-7.

17. Tot T. Cytokeratins 20 and 7 as biomarkers: usefulness in discriminating primary from metastatic adenocarcinoma. Eur J Cancer. 2002;38(6):758-63.

18. Shah RN, Badve S, Papreddy K, Schindler S, Laskin WB, Yeldandi AV. Expression of cytokeratin 20 in mucinous bronchioloalveolar carcinoma. Hum Pathol. 2002;33(9):915-20

19. Hirano T, Auer G, Maeda M, Hagiwara Y, Okada S, Ohira T. Human tissue distribution of TA02, which is homologous with a new type of aspartic protenase, napsin A. Jpn J Canccer Res. 2000;91(10):1015-21.

20. Takano K, Kurosaki I, Minagawa M, Kitami C, Date K, Hatakeyama K. Clinicopathological features of delayed isolated lung metastases after radical pancreatectomy for pancreatic cancer. Jpn J Gastroenterol Surg. 2010:43: $1270-5$

\section{Publisher's Note}

Springer Nature remains neutral with regard to jurisdictional claims in published maps and institutional affiliations.

\section{Submit your manuscript to a SpringerOpen ${ }^{\circ}$ journal and benefit from:}

- Convenient online submission

- Rigorous peer review

- Open access: articles freely available online

- High visibility within the field

- Retaining the copyright to your article

Submit your next manuscript at $\boldsymbol{\nabla}$ springeropen.com 